El Currículo en Acción como Construcción Social y la Contribución Docente con la Transformación Curricular

Rogelis Catillo (pp. 87-102)
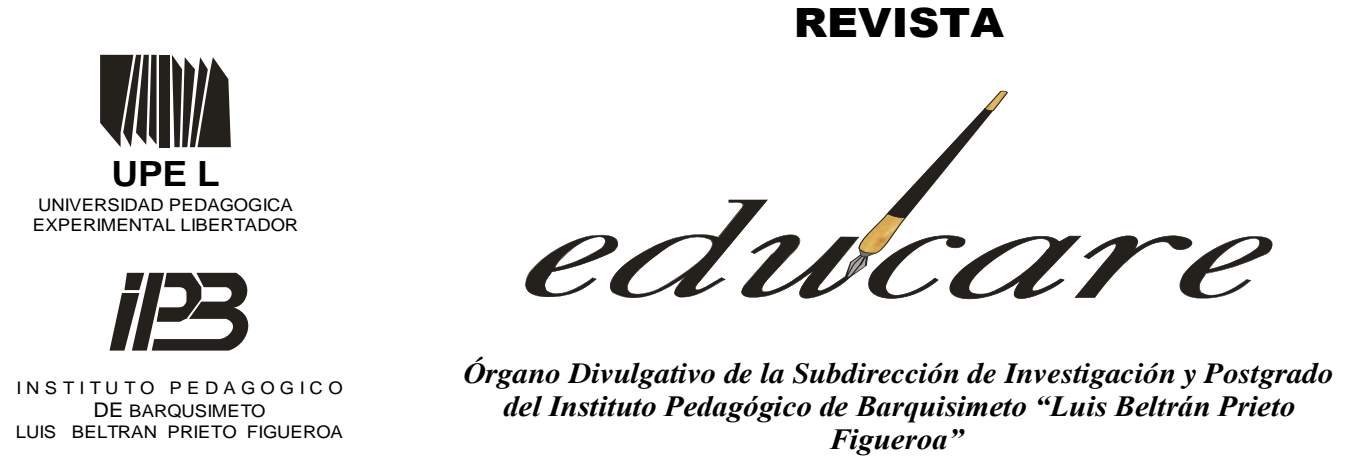

BARQUISIMETO - EDO. LARA - VENEZUELA

NUEVA ETAPA

FORMATO ELECTRÒNICO

DEPOSITO

PPI2O 1002LA3674

LEGAL:

VOLUMEN 19 № 2

ISSN: 2244-7296

MAYO - AGOSTO 2015

EL CURRÍCULO EN ACCIÓN COMO CONSTRUCCIÓN SOCIAL Y LA CONTRIBUCIÓN DOCENTE CON LA TRANSFORMACIÓN CURRICULAR

THE CURRICULUM IN ACTION AS SOCIAL CONSTRUCTION AND THE CONTRIBUTION OF TEACHER TO CURRICULUM TRANSFORMATION

ROgELIS CASTILLO *

"UNIVERSIDAD NACIONAL EXPERIMENTAL SIMÓN RODRÍGUEZ (UNESR) 
El Currículo en Acción como Construcción Social y la Contribución Docente con la Transformación Curricular

Rogelis Catillo (pp. 87-102)

\title{
EL CURRÍCULO EN ACCIÓN COMO CONSTRUCCIÓN SOCIAL Y LA CONTRIBUCIÓN DOCENTE CON LA TRANSFORMACIÓN CURRICULAR
}

\author{
CURRICULUM IN ACTION AS SOCIAL BUILDING AND TEACHING \\ CONTRIBUTION \\ IN CURRICULUM TRANSFORMATION
}

$\underline{E N S A Y O}$

Rogelis Castillo*

UNESR

Recibido:09/06/2015

Aceptado: 20/08/2015

\section{RESUMEN}

El presente ensayo aborda el currículo en acción como construcción social de los docentes ante la transformación curricular de educación básica que propician los entes gubernamentales del Estado Venezolano. La temática se relaciona con hallazgos en una investigación cualitativa relativa a la referida transformación en la Educación Primaria del Estado Portuguesa. Ante un sistema educacional en constante dinámica comunicacional y de cambios sociales que permean la práctica docente, los profesionales construyen socialmente un conjunto de significados en relación con su accionar ante las directrices oficiales. La tesis opuesta sería suponer a los docentes como entes estáticos frente a las incorporaciones de cambios tecnocráticos educacionales, operarios de los diseños curriculares o reproductores acríticos de informaciones. Desde tal perspectiva dialéctica, se genera una mirada diferente de la labor del docente no solo como actor social comunitario sino como profesional reflexivo, albacea de la excelencia académica e innovador en el ambiente de aprendizaje.

Descriptores: currículo en acción, transformación curricular, educación primaria.

\begin{abstract}
This essay addresses the curriculum in action as a social construction of teachers at the basic education curriculum changes that encourage government agencies of the Venezuelan State. The theme is related to qualitative research findings concerning the aforementioned transformation in Primary Education of Portuguesa State. Faced with an educational system in constant communication dynamics and social changes that permeate teaching practice, professionals socially construct a set of meanings in relation to their actions before the official guidelines. The opposite view would assume teachers as static entities against the incorporation of educational technocratic changes, curriculum design operators or uncritical players of information. From the dialectical perspective, a different view of the teacher's work not only as an actor but as a community social reflective, executor of academic excellence and innovative learning environment is generated.
\end{abstract}

Keywords: Curriculum in action, curriculum transformation, primary education

\footnotetext{
* Licenciada en Educación Integral; Magister en Gerencia y Liderazgo en Educación; candidata a Doctora en Educación del Programa Interinstitucional de Doctorado en Educación (PIDE) del convenio UCLAUNEXPO-UPEL, Barquisimeto; Docente Categoría Instructora en la UNESR, Acarigua; Docente III en el Grupo Escolar Bolivariano Durigua, Acarigua. boconorogel@gmail.com
} 


\section{El Currículo en Acción como Construcción Social y la Contribución Docente con la Transformación Curricular \\ Rogelis Catillo (pp. 87-102)}

\section{INTRODUCCIÓN}

En las dos primeras décadas del siglo XXI, Venezuela experimenta medidas oficiales tendentes a lograr la transformación curricular en diversos niveles y modalidades del sistema educativo nacional, regional y local, mediante directrices emanadas de los centros gubernamentales de la educación, concebidas en el contexto del Estado Docente como ente rector delos cambios que -ineludiblemente- están asociados con los mecanismos comunicacionales requeridos por la acción gerencial en la administración pública. Ello implica la participación de actores sociales que se desempeñan en los niveles organizacionales tanto estratégico como de coordinación y operativo, que asumen actitudes en un gradiente que se distribuye entre el franco apoyo y el abierto rechazo.

Las referidas manifestaciones abren espacio para destacar la situación que la literatura especializada denomina currículo en acción, centrado en el docente de aula como actor protagónico que se desenvuelve en el nivel operativo, frecuentemente con pensamiento crítico y accionar estratégico. El aludido currículo en acción, según Gimeno (2007), “es en la práctica real, guiada por los esquemas teóricos y prácticos del profesor, que se concreta en las tareas académicas, que a modo de elementos morales vertebran lo que es la acción pedagógica..." (p.125). Por ello, las voces de los docentes ameritan ser escuchadas e interpretadas en función de la teorización del currículo, dado que sus concepciones, las teorías que practican y los estilos de racionalidad permiten una contribución sustantiva al enriquecimiento de la referida teorización. Es sobre la acción reflexiva como se construye la misma, porque sería iluso pensar que los docentes no manifiestan un estilo de enseñanza y aprendizaje sustentado en su capital cultural, su experiencia profesional y su formación académica, por lo que la idea central del ensayo es plantear cómo los docentes-actores sociales en el nivel de educación primaria viven la transformación del currículo.

En el caso estudiado, los docentes manifiestan constantes desencuentros en relación con los códigos comunicacionales que definen la transformación curricular desde la óptica gubernamental, tanto del nivel de coordinación como del estratégico, por lo que se trata de redirigir las prácticas pedagógicas desde los significados de cada una de ellas mediante la comunicación efectiva, libre de ruidos y distorsiones indeseables. Por ejemplo, es menester 
El Currículo en Acción como Construcción Social y la Contribución Docente con la Transformación Curricular

Rogelis Catillo (pp. 87-102)

la claridad en la concepción de la incorporación de los recursos de aprendizaje, los programas y los temas que comunican los funcionarios del nivel estratégico y de coordinación, en función del éxito de ejecución por parte del docente.

Entre las reflexiones críticas del currículo en acción por parte de los docentes, se destaca la vertiente comunicacional relativa al uso ineficiente de las palabras que, infructuosamente, tratan de definir la transformación curricular, tales como: incorporación de recursos de aprendizaje, modificación de infraestructura, cambios de formatos administrativos, inclusión o eliminación de áreas de trabajo como en el caso del desarrollo endógeno. Asimismo, la articulación de las disciplinas de lengua con cultura, matemática con ciencias naturales o ciencias sociales con ciudadanía y la consideración de elementos como la investigación-acción o la interdisciplinariedad.

En términos de la baja efectividad comunicacional percibida por los docentes-actores sociales, la visión oficial de la transformación curricular se circunscribe a instrucciones relativas a procesos operativos. Ello ilustra el antes aludido desencuentro entre docentes y directivos, que perfilan una realidad ontológicamente emergente o socialmente construida, que amerita su sistematización como ámbito de estudio con enfoque cualitativo, lo cual dio pie a su investigación formal sustentadora de una tesis de doctorado, por lo que este ensayo se sustenta en hallazgos generados a partir de las construcciones sociales de los docentes de educación primaria, interpretados mediante la triangulación cualitativa con los referentes teóricos relacionados con sus testimonios y de la hermenéutica ejercida por la autora de la investigación (Castillo, 2015).

\section{EL CURRÍCULO EN ACCIÓN DESDE LA ÓPTICA DE LOS REFERENTES TEÓRICOS Y LA CONTRIBUCIÓN DOCENTE CON LA TRANSFORMACIÓN CURRICULAR DE EDUCACIÓN BÁSICA}

En esta sección se pasa revista, en primer lugar, al constructo currículo en acción desde la óptica de los referentes teóricos y en segundo lugar su concreción a través de la interpretación de los testimonios de los docentes-actores sociales acerca de su contribución a la transformación curricular de educación básica, representada por tres líneas orientadoras 
El Currículo en Acción como Construcción Social y la Contribución Docente con la Transformación Curricular

Rogelis Catillo (pp. 87-102)

que son: El currículo en acción responde a las dimensiones del docente en relación con el ser, la racionalidad práctica y la intrepidez pedagógica; El currículo en acción permite la renovación del saber pedagógico en la educación primaria; El currículo en acción considera la visión holística entre contenido, pedagogía, didáctica y contextualización para una transformación de educación básica.

\section{El Currículo en Acción desde la Óptica de los Referentes Teóricos}

El Currículo comprende una serie de principios que se originan de las prácticas del docente y de la reflexividad de éstos en el contexto escuela-comunidad. En tal sentido, Gimeno (ob. cit) manifiesta que "el curriculum significa también que su construcción no puede entenderse separada de las condiciones reales de su desarrollo" (p.23). Es decir, el currículo en acción se conceptualiza por lo que las voces de los docentes íntimamente consustanciados con su realidad, agregan a los enunciados teóricos.

En el mismo orden de ideas, la transformación curricular dependerá de la profundidad de los significados atribuidos a las acciones de los docentes, dado que se considera la reflexión como el inicio epistémico del currículo no limitado a la construcción de la normativa, sino a la capacidad de generarlo desde la visión holística de las prácticas docentes concebidas como la teorización, aunada a la coexistencia de la racionalidad técnica y práctica.

Un elemento importante entre las múltiples caras de la situación, es la percepción poco participativa de la transformación curricular señalada por los docentes, dado que los miembros del nivel de coordinación etiquetan sus directrices como Lineamientos del Ministerio de obligatorio cumplimiento. Por tanto, los proyectos y programas devienen en herramientas percibidas como elementos de enfoque tecnocrático de la educación, en ocasiones hiperreales en términos del simulacro descrito por Baudrillard (1978). En este sentido, Posner (2005) desarrolla la idea del currículo con encuadre ilusorio:

Los factores de encuadre son engañosos. Algunos son relativamente fáciles de manipular. Se pueden comprar microscopios, ordenar nuevos libros de texto, pueden ser comparados, modularizar los programas, abolir los grupos por capacidades, combinar los niveles de grado, asignar profesores a equipos e incluso quitar las paredes. Pero ninguno de esos cambios por sí mismo constituye un cambio de un currículo (p. 223). 


\section{El Currículo en Acción como Construcción Social y la Contribución Docente con la Transformación Curricular \\ Rogelis Catillo (pp. 87-102)}

Desde la óptica de este autor, es posible identificar eventos hiperreales asociados con la operatividad de algunos cambios prescritos en el aula. El ser y hacer del docente puede ser entendido como una expresión del currículo en acción que manifiestan estos profesionales desde el principio unitario docente de sus saberes, no para estandarizar sino para comprender e internalizar el uso adecuado de los códigos curriculares.

Por otra parte, el centro de la educación son los estudiantes, idea que se extiende en una preocupación e inquietud de los docentes y, ¿por qué no?, de la comunidad en general. Consecuentemente, las directrices operativas y no consensuadas, significarían alejar el cómo debe trabajar el docente de aula con los estudiantes en actitud constructivista y no bajo el peso tradicional desde reminiscencias de tendencias gerenciales fayolianas. Es decir, se trata de una dialéctica orientada a rescatar la esencia de ser docente con la reflexión del currículo en acción como forma de supervivencia profesional para los educadores.

Por lo antes señalado, cabe referir que Porlán y Rivero (1998) coinciden con Sacristán (ob. cit) sobre el carácter sinérgico del desarrollo del currículo:

No se trata, por tanto, de transmitir directamente los contenidos académicos que, desde la perspectiva del formador, puedan ser relevantes, sino de diseñar una respuesta curricular al problema seleccionado, tomando en consideración informaciones provenientes del saber académico y de la experiencia práctica, ajustadas al nivel de desarrollo de los profesores implicados" (p.170).

De acuerdo con los planteamientos anteriores sobre la concepción tecnicista, los docentes de educación primaria manifiestan sus prácticas educacionales desde una postura reflexiva ante las incorporaciones o modificaciones del currículo oficial, por lo que emerge desde las bases un currículo en acción, cuya fundamentación epistemológica coincide con Gimeno (ob.cit.) al mencionar que: "las tareas definen las situaciones prácticas sobre las que, en forma inmediata, el profesor puede y tiene que reflexionar. Son esquemas de acción en los que puede encontrarse supuestos muy diversos que justifiquen la práctica" (p.320). De esta manera el currículo en acción genera conocimiento que contribuye a teorizar desde las prácticas y su relación con los postulados curriculares.

Uno de estos postulados es argumentado por Gimeno (ob. cit) en relación con las prácticas del curriculum: "la enseñanza y el curriculum han de concebirse como proyecto de investigación en la acción, ya que son en sí problemáticos. Aprovechar todos esos 


\section{El Currículo en Acción como Construcción Social y la Contribución Docente con la Transformación Curricular \\ Rogelis Catillo (pp. 87-102)}

resquicios es una forma de evitar la tendencia reproductora de la educación y del curriculum" (p. 213). Un currículo sin teorización se transformaría en meras prácticas reproductoras de los elementos curriculares. Sin embargo, la investigación socioconstruccionista sobre la cual se basa este ensayo (Castillo, ob. cit), permitió identificar una serie de aspectos teórico-idiográficos que emergen de las transacciones intersubjetivas entre los docentes-actores sociales, mediante la interpretación cualitativa en torno a la teorización del currículo desde la concatenación de la acción reflexiva y de sus postulados académicos, con particular atención a la pertinencia social y a la contextualización con sentido holístico.

Las situaciones consideradas tienen fundamentos asociados con lo expuesto por Gimeno y Pérez (2008) cuando señalan que: “...los problemas de la práctica social no pueden reducirse a cuestiones meramente instrumentales, donde la tarea del profesional se concreta en la acertada elección de medios y procedimientos y en la competente y rigurosa aplicación de los mismos" (p. 407). Ello implica que limitarse al uso de los recursos pudiera convertirse en una ritualización administrativa al proceder por uso y costumbre para el proceso didáctico, así como el confinamiento a una guía acrítica para dirigir la clase, lo cual es divergente de la reflexividad de los protagonistas del currículo en acción.

Adicionalmente, es menester puntualizar que en el currículo en acción, según Gimeno (ob. cit), se concatenan esquemas prácticos con la profesionalidad del docente como el sujeto profundizador de la teorización desde las prácticas reflexivas en las aulas. Por ende, el currículo va más allá de las prescripciones administrativas y el fraccionamiento de saberes por los programas y planes, de igual modo que trasciende la discutible transmisión de contenidos académicos, en procura de la interrelación entre la teoría y la práctica curricular.

En el mismo sentido, Kemmis (2008) explicita que: “el problema central de la teoría del currículum debe ser entendido como el doble problema de las relaciones entre teoría y la práctica, por un lado, y el de las relaciones entre educación y sociedad, por otro" (p.30). Por tanto, el currículo en acción de los docentes de educación primaria implica la necesidad de operativizar el currículo desde el accionar docente con fuente epistémica, es decir, teórico-práctica con tendencias de reflexiones críticas y sentido de contextualización. 


\section{El Currículo en Acción como Construcción Social y la Contribución Docente con la Transformación Curricular \\ Rogelis Catillo (pp. 87-102)}

En razón de lo expuesto, la investigación realizada por Castillo permitió visualizar que los planteamientos teóricos del currículo coinciden en buena medida con las prácticas de los docentes protagonistas de la misma. Aquí emerge un escenario propicio para la proyección de un significado del currículo que no solo dará luces a la educación básica sino a la universitaria, de manera integral y no en términos de un currículo fraccionado.

El asunto es, como plantean Manning, Manning y Long (2000) una especie de relación simbiótica entre la inmersión temática y el currículo en acción, al argumentar que: "la inmersión temática nos permite poner en práctica nuestras ideas acerca de la enseñanza y el aprendizaje" (p.21). Es decir, la operatividad del currículo en acción se manifiesta y se interrelaciona con el cómo se pregunta acerca de las áreas académicas vinculadas con el mismo y su imbricación con las dimensiones contextuales intra y extraescolares inherentes a la relevancia y pertinencia social. Es decir, la reflexividad del docente como protagonista del currículo en acción debe ser de mirada profunda y envolvente de la teoría y la práctica.

\section{La Contribución del Docente con la Transformación Curricular de la Educación}

\section{Básica}

Una de las consideraciones emergentes en la investigación de Castillo (ob. cit), fue la manera como los docentes manifestaron sus relaciones epistemológicas con el currículo oficial. Entre ellas se presentó la interrelación docente-estudiante con los recursos de aprendizaje que, a su juicio, debe ser dinámica y reflexiva sin estandarizar los procesos. Del impacto de esa interrelación afloró un choque conceptual entre lo que se concibe como currículo y sus diferencias con la concepción del diseño curricular. A través de las prácticas profesionales cotidianas, los docentes de educación primaria reflexionaron acerca de que uno de los vacíos de la transformación curricular era pretender trabajar un currículo oficial como si se tratara del diseño curricular, sin diferenciar el uno del otro y pretender visualizarlo como una guía metodológica que conduce a una ritualización de los procesos.

Otro de los obstáculos epistemológicos de la transformación curricular, desde la óptica de los docentes, consistió en la discrepancia con la tendencia a pretender ver los contenidos solamente desde lo local, cuando los mismos estudiantes presentan inquietudes y fascinación de proyectarse hacia la mundialización. Ante ello, emerge la posibilidad de 
El Currículo en Acción como Construcción Social y la Contribución Docente con la Transformación Curricular

Rogelis Catillo (pp. 87-102)

una dialéctica docentes-estudiantes propicia para que sus discursos integren a la comunidad a un currículo en acción, sobre la base de los enunciados registrados en los siguientes párrafos.

\section{El Currículo en Acción responde a las Dimensiones del Docente en relación con el Ser,}

\section{la Racionalidad Práctica y la Intrepidez Pedagógica}

De acuerdo con las versiones de los actores sociales en el estudio de Castillo (ob. cit), en el momento de asumir vocacionalmente la carrera docente como profesión, ya es considerada un compromiso intrínseco con la transformación curricular que la convierte en foco permanente de reflexividad. Por ello, sin distinción de los años de servicio de un docente, todos contribuyen de alguna manera a la construcción, deconstrucción y reconstrucción de las prácticas educacionales. No obstante, es común en los discursos tanto de gerentes como entre colegas, el señalamiento de que el letargo en el cambio educativo se debe a la falta de vocación de los docentes en ejercicio, sin considerar otros factores.

En ese sentido, algunos estereotipos expresados por los funcionarios del nivel de coordinación para describir a los docentes de aula se limitan a los deseos o intenciones del gerente que pretende dar directrices sin ocuparse de convencer a sus colegas de las bondades de sus ideas. En consecuencia, si los docentes no cumplen con lineamientos tales como ser creativos, innovadores, investigadores, flexibles, líderes, orientadores, psicólogos, gerentes, segundos padres y hasta médicos, los miembros del nivel de coordinación los etiquetan como carentes de vocación o resistentes a los cambios.

En oposición a la anterior percepción, los docentes consideran que los estereotipos son sustituidos por el currículo en acción al priorizar que la construcción social de la transformación curricular se gesta en las relaciones con el otro, el diálogo, el saber y hacer docente durante el proceso de socialización de la misma. Ellos plantean resignificar la profesión docente desde el accionar de sus prácticas al ser agentes de la transformación curricular, por lo que no se trata de estar interesados en quien nomina alumnos en vez de estudiantes o viceversa para juzgar el desempeño del educador, sino que es importante reflexionar el significado de ser docente crítico o contrariamente convertirse en operarios de las prescripciones administrativas. En este caso cabe, sobre la base de los testimonios de 


\section{El Currículo en Acción como Construcción Social y la Contribución Docente con la Transformación Curricular \\ Rogelis Catillo (pp. 87-102)}

los actores sociales, el término de educador que se encarga, con racionalidad práctica, de extraer y cultivar la semilla de esperanza de la transformación curricular (Castillo, ob. cit).

Las contribuciones de los docentes para un currículo en acción se expresan entre el encuentro personal, el repensar y difundir códigos acordes con la educación, instrucción, escuela y una cultura curricular como el principio de ser personas progresivamente más humanas. En este orden de ideas Stenhouse (1998) plantea que "El currículo no es la intención o la prescripción, sino lo que acontece en situaciones reales. No es la aspiración, sino el logro" (p.26). Es decir, el escenario de las raíces del razonamiento dialéctico planteadas por Kemmis (ob. cit) en relación con la concepción de un currículo es la unidad de los opuestos a través de la comprensión del cómo se relacionan entre sí.

De allí que el currículo en acción sea el encuentro del docente consigo mismo. Es desde su cotidianidad lo que permite la semilla de la transformación curricular en contraposición con la multiplicidad asistemática de la burocracia, a veces sin sentido teórico-práctico, en el aula y su proyección hacia la comunidad, tal como lo recuerda Flórez (2005) "el currículo, entonces, se configura desde el exterior hacia el interior de la persona, en el análisis de la sociedad y la cultura, detectando símbolos, mitos, lenguajes, valores y relaciones sociales para transformar la educación” (p.296).

En concordancia con lo planteado, la dimensión de la racionalidad práctica es considerada la fuente epistémica del currículo en acción ante un escenario de múltiples prescripciones administrativas sin tratar de limitar éstas a lineamientos ministeriales, sino de reflexionarlas sobre la base de las prácticas pedagógicas. Expresado de otro modo por Gimeno y Pérez (ob. cit) no es suficiente aplicar una serie de programas o proyectos de enseñanza sin comprender los fenómenos sociales que implica la ejecución de un currículo.

Otra de las dimensiones del currículo en acción se refiere a la intrepidez pedagógica del docente, la cual representa a quienes encaran una serie de actividades escolares y el cómo se interrelacionan con los estudiantes y sus jefes inmediatos. Así como los docentesgerentes comparten sus experiencias de socialización de la transformación curricular con otros gerentes, los docentes de aula realizan prácticas cotidianas reflexivas entre par

Lo antes expuesto concuerda con el planteamiento de Gimeno (ob. cit) al aducir que: "Los propios efectos educativos dependen de la interacción compleja de todos los aspectos 
El Currículo en Acción como Construcción Social y la Contribución Docente con la Transformación Curricular

Rogelis Catillo (pp. 87-102)

que se entrecruzan en las situaciones de enseñanza: tipos de actividad metodológica, aspectos materiales de la situación, estilo del profesor, relaciones sociales, contenidos culturales, etc." (pp. 242-243). Consecuentemente, cabe destacar que por estas condiciones de permanente interacción con el otro, el currículo en acción refleja tres dimensiones esenciales del docente: ser, racionalidad práctica e intrepidez pedagógica.

\section{El Currículo en Acción permite la Renovación del Saber Pedagógico en la Educación}

\section{Básica}

Otra de las contribuciones del estudio de Castillo (ob. cit), revela la complejidad de la transformación curricular que tiene un significado que rebasa las prescripciones asumidas desde la connotación de burocracia administrativa o la incorporación de recursos materiales como el cambio radical en la Educación Básica, al reflexionar sobre la acción. El mismo reivindica la posibilidad de sentirse libres para aportar los propios puntos de vista al momento de compartir con los estudiantes, así como considerar a la transformación curricular como el encuentro de sí mismos como personas pensantes y actuantes.

La renovación del saber pedagógico en la Educación Básica se refiere no solo a resolver problemas de cálculo, lectura, escritura, valores ciudadanos o ambientalistas, sino el qué, por qué y cómo hacer, para resignificar la pedagogía considerada sin limitaciones a una simple ejecución metodológica. El saber pedagógico, más allá de simulaciones curriculares, es comprender los fenómenos sociales sin buscar culpas de las debilidades en una determinada institución educacional y proceder de la manera más constructiva posible. Se trata de considerar la transformación curricular desde el saber pedagógico como el espacio de aprender, desaprender y reaprender los modelos teóricos del proceso de enseñanza y aprendizaje. Es tener conciencia ecológica del ambiente de aprendizaje durante la planeación estratégica de lo operativo que implica un currículo.

En el sentido de lo expuesto, Echeverri (2003) enuncia la consustancialidad de la lengua y el pensamiento al sistematizar los saberes pedagógicos:

El lenguaje es retomado por el maestro no como un objeto o cosa sino como un discurso en el que se entrelazan usos, estrategias y teorizaciones, que garantizan al maestro y al alumno su residencia en el lenguaje como morada de su práctica pedagógica (p.176). 
El Currículo en Acción como Construcción Social y la Contribución Docente con la Transformación Curricular

Rogelis Catillo (pp. 87-102)

Según los planteamientos anteriores, es permisible que un currículo en acción renueve el saber pedagógico hacia una conexión más profunda con el proceso educativo, por medio de las nociones de educación, sociedad, relación con el currículo, búsqueda permanente del saber y metacomunicación, así como la metateoría del currículo. Por ello cabe plantear la concepción clásica de Wittgenstein (1999) sobre el juego de palabras para argumentar la premisa del currículo en acción en función de la transformación del mismo que se perfila hacia la palabra-acción. No se trata del significado de la palabra en un diccionario o por moda, sino por el uso que le dan los docentes cuando comunican aspectos sobre la transformación curricular. He allí la competencia pedagógica más allá de la metodología de los procesos.

En sintonía con los planteamientos anteriores cabe citar a Morin (2002), quien manifiesta: "En la educación se trata de transformar la información en conocimiento, de transformar el conocimiento en sapiencia" (p. 49). En otras palabras, la ruta del currículo en acción manifestado por los docentes de educación primaria participantes en el estudio de Castillo (ob. cit), permite reivindicar un espacio para la renovación del saber pedagógico.

\section{El Currículo en Acción considera la Visión Holística entre Contenido, Pedagogía,}

\section{Didáctica y la Contextualización para una Transformación en Educación Básica}

La tercera contribución de la investigación de Castillo (ob. cit), consiste en la concepción de la transformación curricular ante las simulaciones en las organizaciones educacionales durante la socialización entre docentes ante los vacíos de la interpretación de la teoría curricular que permiten el desencuentro de los currícula (el oficial y el presentado) y el afloramiento del currículo en acción, el cual se asume desde la concepción de visión holística entre contenido, pedagogía, didáctica y la contextualización para una transformación curricular en Educación Básica.

El currículo en acción permea el principio holístico entre contenido referido a las áreas académicas del diseño establecido y la pedagogía como el saber internalizar y reflexionar la teoría curricular, además de la didáctica como el medio para operativizar la pedagogía y todo ello para contextualizar según las concepciones de la teoría curricular: 


\section{El Currículo en Acción como Construcción Social y la Contribución Docente con la Transformación Curricular \\ Rogelis Catillo (pp. 87-102)}

teorías de aprendizaje, la antropología de la educación, los avances de la ciencia y la sociedad.

En ese sentido, Gimeno (s/f) señala que "Buena parte de la proyección de la teoría del aprendizaje en la teoría pedagógica y en la práctica educativa se ha realizado de una forma asistemática y acrítica, sin establecer el filtrado epistemológico” (p.153-154). Por ello, es menester concebir la transformación curricular como el encuentro del saber docente desde una visión holística de cada proceso. Además, una práctica pedagógica no debe estar aislada de la teoría curricular ni vista como simulaciones de cambio o como reproducción social acrítica de la información.

La responsabilidad de la transmisión de la información como fuente primordial se materializa a través de los recursos técnicos que se utilizan para etiquetar cambios que son interesantes y pertinentes, pero limitados para la transformación curricular en un hacer tecnicista que no solo se codifica y decodifica con los medios tecnológicos, sino que las informaciones curriculares son de carácter prescriptivo. Ejemplos de ello, están dados por la computadora canaima, el libro bicentenario y la revista tricolor, presentados como parte de la prescripción administrativa para el trabajo docente y no como recursos de aprendizaje.

En resonancia con los anteriores cuestionamientos al tratamiento inadecuado de los recursos para el aprendizaje que se utilizan para etiquetar cambios, es prudente citar a Pérez (2012), quien expresa que:

La imagen, la pantalla, desarrolla sistemas perceptivos, procesos mentales y respuestas distintas que la lectura, privilegia la percepción sobre la abstracción, lo sensitivo sobre lo conceptual, la forma sobre el contenido, el espectáculo sobre la reflexión, lo concreto sobre lo abstracto (p. 57-58).

El uso inadecuado de los mismos, en vez de ser recursos para un aprendizaje significativo se convierten en un sinsentido curricular, realidades encontradas entre simulaciones y a la vez desvinculadas de la teoría. Esto genera una imagen instrumental de las prácticas pedagógicas así como la reproducción social que forma vacíos y hasta incongruencias con lo declarado en el currículo prescrito y en el presentado por el gerente.

Por otra parte, sí la pedagogía en educación primaria es crítica, liberadora y están ausentes las teorías del aprendizaje principalmente la de Piaget, por no tener planteamientos 


\section{El Currículo en Acción como Construcción Social y la Contribución Docente con la Transformación Curricular \\ Rogelis Catillo (pp. 87-102)}

propios con los ideales sociocríticos, entonces, por qué insertar esa teoría psicogenética en el currículo presentado por el gerente, según documento oficial, como una innovación no asociada con el prenombrado autor. No obstante, Pérez (s/f) afirma que "La teoría de Piaget, precisamente por su carácter genético y por su carácter epistemológico, ofrece una plataforma conceptual para afrontar, desde una racionalidad revolucionaria e integradora, el dualismo forma-fondo, procesos-resultados, métodos-contenidos" (p.126).

De acuerdo con las interpretaciones de los testimonios de los actores sociales, se trata, de reflexionar desde una teoría curricular que se encuentra en la acción de los docentes para no limitarse a concepciones que descontextualizan la pedagogía y la didáctica de un diseño curricular.

\section{REFLEXIONES FINALES}

El currículo en acción es el espacio de reflexión sobre las incorporaciones de los recursos de aprendizaje, programas y proyectos educacionales desde un estilo ecológico al implicar personas que intervienen durante los procesos y son ellas las que permiten la construcción de una transformación curricular. Entonces, antes de trabajar por la integración entre escuela y comunidad, se deben internalizar los procesos entre docente, estudiante y gerente. Es decir, es necesario identificar los códigos curriculares de los docentes con funciones gerenciales y de aula en función de proyectarse a la comunidad.

El currículo en acción se genera desde la reflexión de las prácticas pedagógicas entre los docentes y los estudiantes para comprender, profundizar e internalizar cada elemento de la educación. Se refiere a un ambiente complejo de permanente reflexión entre las actividades escolares, las relaciones entre los actores, sus percepciones, emociones, creencias, mitos y hasta realidades sobre lo que implica una racionalidad práctica de los procesos.

Por lo tanto, la Educación Básica tiene un renovado horizonte representado por el currículo en acción de los docentes que siguen inquietos y constructivos para un punto de encuentro entre los agentes educacionales en relación con la transformación curricular. Y es que los docentes no se limitan a ser operadores de información curricular sino seres 


\section{El Currículo en Acción como Construcción Social y la Contribución Docente con la Transformación Curricular \\ Rogelis Catillo (pp. 87-102)}

humanos que siembran, construyen, riegan y contribuyen con la transformación de la educación básica desde la conciencia ecológica, en un ecosistema con condiciones aún adversas para el reencuentro de las raíces de ser docentes.

Finalmente, la investigación socioconstruccionista desarrollada por Castillo (ob. cit) genera una visión diferente y pertinente de la labor del docente no solo como actor social comunitario sino como profesional reflexivo, albacea de la excelencia académica e innovador en el ambiente de aprendizaje.

\section{REFERENCIAS}

Baudrillard, J. (1978). Cultura y simulacro. Barcelona, España: Editorial Kairós.

Castillo, R. (2015). La transformación curricular en el nivel de educación primaria como visión socioconstruccionista de los docentes. Tesis de doctorado no publicada. Barquisimeto: Universidad Pedagógica Experimental Libertador.

Echeverri, J. (2003). El lugar de la pedagogía dentro de las ciencias de la educación. En Zuluaga, O., Echeverri, A., Martínez, A., Quiceno H., Sáenz, J. y Álvarez, A. (Comp.). (2003). Pedagogía y epistemología (pp.127-184). Colombia: Magisterio.

Flórez Ochoa, R. (2005). Pedagogía del conocimiento (2a. ed.). Bogotá: McGraw-Hill.

Gimeno, J. (2007). El curriculum: una reflexión sobre la práctica. (9a. ed.). España: Morata.

Gimeno, J. (s/f). La integración de la teoría del aprendizaje en la teoría y práctica de la enseñanza. En Graham, I., Hunter, I., Brainerd, C., Moreno, M., Benlloch, M. y Pérez Gómez, A. (Comp.). (s/f). Teorías del aprendizaje social II. Piaget y la pedagogía operatoria. Cuadernos de educación no 169 (pp.145-179). Caracas: Laboratorio Educativo.

Gimeno, J. y Pérez, A. (2008). Comprender y transformar la enseñanza (12a. ed.). España: Morata.

Kemmis, S. (2008). El currículum: Más allá de la teoría de la reproducción (4a. ed.). España: Morata.

Manning, M., Manning, G. y Long, R. (2000). Inmersión temática: el currículo basado en la indagación para los primeros años y años intermedios de la escuela elemental. España: Gedisa.

Morin, E. (2002). La cabeza bien puesta. Buenos Aires: Nueva Visión.

Pérez, A. (2012). Educarse en la era digital. España: Morata. 
El Currículo en Acción como Construcción Social y la Contribución Docente con la Transformación Curricular

Rogelis Catillo (pp. 87-102)

Pérez, A. (s/f). Piaget y los contenidos del currículo. En Graham, I., Hunter, I., Brainerd, C., Moreno, M., Benlloch, M. y Gimeno, J. (Comp.). (s/f). Teorías del aprendizaje social II. Piaget y la pedagogía operatoria. Cuadernos de educación n n 169 (pp.123143). Caracas: Laboratorio Educativo.

Posner, G. (2005). Análisis de currículo. Docente del siglo XXI. Colombia: McGraw-Hill.

Schön, D. (1987). La formación de profesionales reflexivos: Hacia un nuevo diseño de la enseñanza y el aprendizaje en las profesiones. España: Paidós.

Stenhouse, L. (1998). Investigación y desarrollo del curriculum (4a. ed.). España: Morata.

Wittgenstein, L. (1999). Investigaciones filosóficas. España: ALTAYA. 